\title{
BMJ Open Oxford Understanding Relationships, Sex, Power, Abuse and Consent Experiences (OUR SPACE) cross- sectional survey: a study protocol
}

\author{
Bridget Steele (D) , ${ }^{1}$ Michelle Degli Esposti (D) , ${ }^{1}$ Pete Mandeville, ${ }^{2}$ Gillian Hamnett, ${ }^{3}$ \\ Elizabeth Nye, ${ }^{1}$ David K Humphreys ${ }^{1}$
}

To cite: Steele B, Degli Esposti M, Mandeville P, et al. Oxford Understanding Relationships, Sex, Power, Abuse and Consent Experiences (OUR SPACE) cross-sectional survey: a study protocol. BMJ Open 2021;11:e051826. doi:10.1136/ bmjopen-2021-051826

- Prepublication history for this paper is available online. To view these files, please visit the journal online (http://dx.doi. org/10.1136/bmjopen-2021051826).

Received 29 March 2021 Accepted 14 0ctober 2021

Check for updates

(C) Author(s) (or their employer(s)) 2021. Re-use permitted under CC BY-NC. No commercial re-use. See rights and permissions. Published by BMJ.

${ }^{1}$ Department of Social Policy and Intervention, University of Oxford, Oxford, UK

${ }^{2}$ Student Welfare Service Lead, University of Oxford, Oxford, UK ${ }^{3}$ Director of Student Welfare and Support Services, University of Oxford, Oxford, UK

Correspondence to

Bridget Steele;

bridget.steele@spi.ox.ac.uk

\section{ABSTRACT}

Introduction Sexual violence among higher education students is a public health concern, threatening the general safety of students, often with significant physical and mental health implications for victims. Establishing the prevalence estimates of sexual violence at higher education institutions (HEls) is essential for designing and resourcing responses to sexual violence, including monitoring the effectiveness of prevention initiatives and institutional programmes. Yet, to date, there have been no rigorous studies assessing prevalence of sexual violence at HEls in the UK.

Methods and analysis Informed by guidance from Universities UK, the University of Oxford administration and the related student advocacy groups working within the University, Oxford Understanding Relationships, Sex, Power, Abuse and Consent Experiences is a cross-sectional survey of all undergraduate and graduate students over the age of 18 enrolled at the University of Oxford, UK. The survey design uses a complete sampling approach and measures adapted from previous campus climate surveys in the USA as well as the Sexual Experiences Survey (USA). The analysis will estimate the prevalence of sexual harassment and sexual violence perpetration and victimisation, and will examine whether ethnicity, gender identity, and sexual orientation are associated with these primary outcomes.

Ethics and dissemination Ethical approval was obtained by the Social Sciences and Humanities Interdivisional Research Ethics Committee at the University of Oxford which is a subcommittee of the Central University Research Ethics Committee (ref no.: R73805/RE001). The research team will disseminate findings through peerreviewed journal articles and conference presentations. A report cowritten by authors and stakeholders will be shared with Oxford University students.

\section{INTRODUCTION}

Sexual violence among higher education students threatens the safety of young people enrolled in higher educational institutions (HEIs) and impacts their physical and mental health. ${ }^{12}$ While prevalence estimates of sexual violence at HEIs can be difficult to establish, due to inconsistent definitions of
Strengths and limitations of this study

- Stakeholder engagement and involvement throughout the development and implementation of the study.

- Complete sampling of the population.

- Findings will impact prevention and response initiatives at the University of Oxford.

- Cross-sectional survey design, with the potential for longitudinal data collection at the University of Oxford and in other UK higher education institutions.

sexual violence and methodological disparities across studies, prevalence estimates are key to: (1) understanding what resources need to be directed toward sexual violence response and prevention initiatives; (2) monitoring the effectiveness of prevention and response initiatives; and (3) using student voices to inform institutional policies and procedures. ${ }^{3}$ Collecting sexual violence data through surveys is also essential, as underreporting of sexual violence means that formal reports to HEIs or to law enforcement officials are limited in their ability to accurately estimate the scale of the problem. ${ }^{4}$

Sexual violence prevalence surveys at HEIs in the USA estimate that in many institutions over $20 \%$ of women are sexually assaulted while attending an American HEI. $^{2} 35$ Prevalence rates for transgender or gender-nonconforming individuals at US HEIs are even higher, with an estimated $24 \%$ experiencing sexual assault. ${ }^{6}$ These surveys conducted by US colleges and universities have been influential in creating and improving prevention and support services for students within these institutions. ${ }^{3}$ As suggested in Harris et $a l^{7}$, there is a 'growing need for high-quality empirical research' on sexual violence among HEI students outside of the US context; sexual violence among HEI 
students could manifest itself differently across different geographies and societies. ${ }^{7}$

The evidence base on sexual violence prevalence among HEI students in the UK is particularly lacking. To date, no rigorous study has established prevalence of sexual violence at HEIs in the UK context. The most recent available UK estimates are from newspaper surveys that indicate that over half of female students have experienced sexual harassment, and approximately $14 \%$ report serious physical or sexual assault. ${ }^{89}$ The National Union of Students have also carried out research on sexual violence prevalence at UK HEIs. Their Hidden Marks survey was administered in 2009 and 2010 to assess the prevalence and nature of sexual assault and harassment. ${ }^{10}$ This survey found that $14 \%$ of women respondents were survivors of severe sexual assault or physical violence while attending an HEI in the UK. ${ }^{10}$ No subsequent Hidden Marks surveys have been administered and there is no evidence on victims who are men or sexual violence in a non-heteronormative context.

In 2016, Universities UK established a taskforce made up of university administration, student representatives and academics who were encouraged to evaluate the problem of sexual violence using all available evidence. ${ }^{11}$ The resulting 2016 report found that there was 'no comprehensive data available' on sexual violence at universities in the UK. ${ }^{11}$ Universities UK encouraged institutions to have a 'coherent, systematic approach across the whole institution' and suggested collaboration between senior leadership and internal academics to collect high-quality data on prevalence to assess the effectiveness of prevention and response efforts. ${ }^{11} 12$

HEIs have the potential, with their personnel, purpose, and infrastructure, to be at the forefront of engaging in rigorous data collection, knowledge sharing, and implementing innovative collaborative and evidence based interventions within institutional settings. ${ }^{7}$ They are also a 'particularly promising setting for effectively bringing together research and practice'. ${ }^{7}$ Collecting HEI-specific data about the nature and prevalence of sexual violence in the UK context offers practitioners the opportunity to make evidence based decisions about prevention and response programmes and policies. ${ }^{3}$

\section{RESEARCH OBJECTIVES}

There are no existing survey data on the prevalence of sexual violence at the University. The term sexual violence is broad and includes all non-consensual or unwanted sexual acts including harassment, sexual assault and rape.$^{13}$ We aim to conduct a campus climate survey entitled Oxford Understanding Relationships, Sex, Power, Abuse and Consent Experiences-an inclusive cross-sectional study of all undergraduate and graduate students over the age of 18 at the University of Oxford. The aim of this research is to understand the problem of sexual violence among HEI students in the UK to inform data collection and prevention and response initiatives at the University of Oxford and across the sector. To achieve this aim, this study will address following research question: What is the prevalence and nature of sexual violence at the University of Oxford? Specific objectives include:

1. Partner with University of Oxford administration, sexual violence support services and relevant student government and groups to administer the survey.

2. Develop a prevalence estimate for sexual violence among students at a UK HEI.

3. Describe sexual violence perpetration and victimisation by gender identity, sexual orientation, disability and race/ethnicity.

4. Understand student attitudes on consent and sexual violence.

5. Develop a set of standard questions that can be used to evaluate and compare sexual violence at a national level.

\section{METHODS}

\section{Patient and public involvement}

Following suggestions by Universities UK, this research is and will continue to be informed by relevant stakeholders, specifically University of Oxford administration and the student advocacy groups working within the University. ${ }^{11}$ This involved regular meetings with the University's Sexual Harassment and Violence Support Service, the Oxford Student Union, the It Happens Here Campaign, and university communications officials over the course of a year leading up to the survey launch. Further, we presented the survey at a series of collegiate University welfare forums and communications groups. This research has also received support from the University of Oxford's Governance and Planning Office's van Houten Fund. University-wide working groups will bring together interdisciplinary academic specialists, student groups, and university administration to facilitate a whole-institution approach. ${ }^{11}$ These stakeholders will be involved in the development of the survey, the interpretation of the findings, and the communication of the results.

\section{Design and setting}

The survey is a cross-sectional survey based at the University of Oxford, in the Southeast region of the UK. The University of Oxford is a university comprised of 39 colleges that was founded in $1096 .{ }^{14}$ Many students typically live in college accommodation and seek the academic and welfare support offered by their college. Due to the COVID-19 pandemic, many students may not be living in their college communities or in the city of Oxford during the administration of the survey and we will account for this in the questions asked to respondents. There are over 24000 students currently attending the University of Oxford, half of which are undergraduates with the other half being postgraduates. The university is an international environment, with $20 \%$ of undergraduate students and $64 \%$ of all graduate students coming from outside 
of the UK..$^{15}$ According to 2019 data, the University of Oxford population consists of approximately $80 \%$ white students and $20 \%$ black and minority ethnic students. The admitted class of 2019 was made up of $54.4 \%$ women, $9.4 \%$ students with a disability and $87.8 \%$ of students living in 'more advantaged areas'.

\section{Participants}

The population includes all registered undergraduate and postgraduate students (including part-time, visiting and online students), over the age of 18. The sample excludes students under the age of 18 given that they would need parental consent to participate.

\section{Procedure}

The design employs a complete sampling approach. An email with a link to the survey will be sent to the entire sample population. Participants will then be asked to give consent to anonymously participate in the study. The survey will be launched in May 2021. As the University of Oxford academic year consists of three terms (trimesters), the survey was launched during May-July 2021 (the final term) to capture experiences from the entire academic year. Emails will be supplemented by additional online promotional campaigns run through the student government, colleges and university platforms, to raise awareness of the survey, to educate students on the importance of participating in the study, and to encourage participation in the study. The link to complete the survey will be open for 2 months after the launch and students will receive three reminder emails if they have yet to fill out the survey. Therefore, the study collection period will span 2 months from May 2021 to July 2021. Those who complete the survey will indicate if they want to be entered into a lottery to receive a prizes (iPads (3) and $£ 50$ Amazon vouchers (10)).

Qualtrics survey software is the electronic platform used to implement the survey. Students will be able to click on a link included in their recruitment email which will take them to an information and consent page. This page will provide information on the study, the investigators and the intention of the research. Students will be asked to give consent to complete the survey through clicking a box. The survey will take approximately 15-20 min to complete, and students will be able to terminate the survey at any time. Students who participate will be provided with a list of supports and resources before, during and after completing the survey in accordance with ethical guidelines.

\section{Survey measures and outcomes}

Survey measures are adapted from previously conducted campus climate surveys in the US context, specifically the Johns Hopkins University Health and Safety Study from 2017, and psychometric questions from the Sexual Experiences Survey (SES). ${ }^{16}$ The SES has been validated in the US context but has yet to be validated in the UK context. ${ }^{17}$ The questions used are also informed by the available evidence on risk and protective factors for sexual violence in this setting and conversations with practitioners working to prevent and respond to sexual at the University of Oxford. ${ }^{18}$ These stakeholder consultations informed the contextual adaptations (both to the University of Oxford and to the UK more broadly) of the survey. For example, the inclusion of questions that reflect the role that colleges play in the University, asking questions related to institutional response, and asking questions about type of secondary school attended. We excluded questions used to assess sexual violence programming that does not exist in the UK context. We established content validity through asking members from the target population and professionals in the field to assess the survey for relevance, comprehensiveness and comprehensibility. This was done through regular focus group discussions throughout the survey development process and through having members from the target group pilot the survey.

The survey, outlined in table 1 focuses on four key areas: 1. Sociodemographic characteristics: the survey asks about participants' gender identity, sexual orientation, race/ethnicity and parental education. Participants are also asked to identify if they are living with a disability and they are asked where they are living during the academic year.

2. University climate: participants are asked about their beliefs pertaining to statements about consent and rape-myths, whether or not they are aware of university support services for victims of sexual violence, and their bystander behaviour.

3. Sexual harassment victimisation and perpetration: participants will have the opportunity to indicate if they experienced or perpetrated a series of sexual harassment acts in the past year. Participants who identify that they have experienced or perpetrated an act will be directed to a series of related follow-up questions pertaining to the incident.

4. Sexual violence victimisation and perpetration: victimisation will be measured using self-reported experiences of (1) attempted sexual violence and (2) completed sexual violence. An adapted version of The Sexual Experiences-Short Form Victimisation ${ }^{16}$ will measure non-consensual sexual contact and sexual violence. Adaptions were made based on guidance from to create more inclusive wording that can incorporate female perpetrated sexual violence against men and that can include assaults that students, think, but are not sure happened, due to alcohol or drug consumption. ${ }^{19}$ Perpetration will be measured using selfreported experiences of (1) attempted sexual violence and (2) completed sexual violence. Perpetration will be measured using an adapted version of the Sexual Experiences-Short Form Perpetration. ${ }^{16}$ To improve recall reliability, participants will be asked about sexual violence in the past calendar year. ${ }^{20}$ If an incident of sexual violence was experienced or perpetrated, then students will be asked follow-up questions related to this incident including questions on who was involved, 
Table 1 Survey content

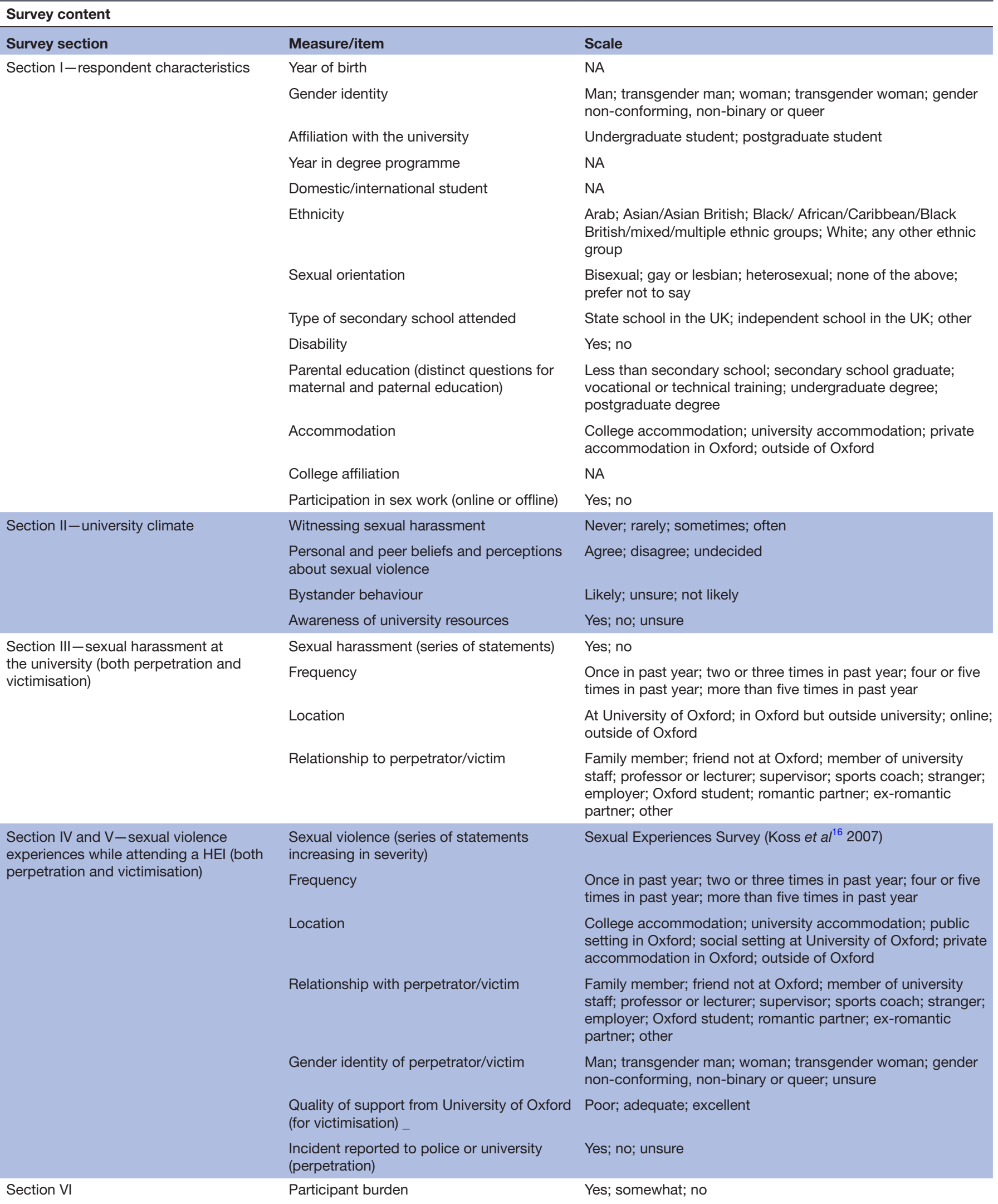

HEl, higher educational institution. 
the relationship with the victim or perpetrator, the location of the violence, and the effectiveness of supports accessed or consequences for the act.

\section{Power calculations}

Following guidance from Hajian-Tilaki, ${ }^{21}$ we calculated our desired sample size, relying on an estimated prevalence rate and a margin of error of $1 \%$. Given that previous, rigorous, research from the USA estimates the prevalence of sexual violence among women attending HEIs students to be approximately $20 \%$, we calculate that we require a sample size of at least 1537 respondents to form an adequate sample. ${ }^{15}$ Decisions will be made about which subgroups have enough power to be examined based on the number of respondents in each sociodemographic subgroup.

\section{Planned analysis}

\section{Prevalence of sexual violence}

We will calculate descriptive frequencies to estimate the proportion of the University of Oxford student population to have experienced any act of sexual violence or harassment in the reporting period (1 year). Specifically, we will calculate the 1-year period prevalence for each of the primary outcomes of victimisation and perpetration of sexual violence and sexual harassment, treating each outcome as a binary variable. ${ }^{22}$ For example, for victimisation of sexual violence, those who did report experiencing sexual violence victimisation will be coded as 0 , while those who reported one or more experiences of sexual violence victimisation will be coded as 1 . Occurrences will be stratified by sociodemographic groups (eg, ethnicity, gender identity and sexual orientation), using cross-tabulations and $\chi^{2}$ statistics to compare and test for significant differences across subgroups.

\section{University climate and bystander behaviour}

We will calculate average scores for each question to summarise respondents' personal attitudes and beliefs on a series of statements about sexual harassment and violence, and their perception of their peers' attitudes and beliefs about the same statements (response options are 'agree'; 'unsure'; 'disagree'). Similar average scores will be derived on a series of statements assessing for participants' confidence and willingness to engage in effective bystander behaviour (response options are 'likely'; unsure; 'not likely'). Averages will be stratified by sociodemographic groups (eg, ethnicity, gender identity and sexual orientation), using cross-tabulations and $\chi^{2}$ statistics to compare and test for significant differences across subgroups.

\section{Associations with sociodemographic characteristics}

We will examine whether ethnicity, gender identity and sexual orientation are associated with the primary outcomes (victimisation and perpetration of sexual harassment and sexual violence).${ }^{18}$ We will run a series of bivariate and multivariate logistic regression analyses to examine whether specific sociodemographic characteristics (eg, identifying as woman) are associated with increased odds of experiencing or perpetrating any act of sexual violence or sexual harassment. We will first assess unique one to one associations between sociodemographic variables and the primary outcomes. Then we will conduct unadjusted multivariate analyses for each sociodemographic variable separately, in relation to all primary outcomes. We will then run adjusted multivariate analyses to examine the associations between sociodemographic variables and primary outcomes. This will allow us to understand the relative contribution of risk of each sociodemographic characteristic (eg, gender) on each outcome while controlling for other characteristics (eg, sexual orientation and race/ethnicity).

\section{Clustering and co-occurrence of sexual violence}

Frequencies will also be derived for multiple types of sexual violence experienced (ie, counts), both within and across primary outcomes to describe the clustering occurrences of sexual violence within individuals and examine associations across victimisation and perpetration. We will use cross-tabulations to describe the number of occurrences of each primary outcome (eg, number of times a participant reported experiencing an act of sexual violence) and co-occurrences across outcomes (eg, whether they reported both experiencing and perpetrating an act of sexual violence).

\section{Severity and location}

We will assess the severity of the violence reported and the location where the violence occurred. The survey asks a series of questions regarding sexual harassment and sexual violence victimisation and perpetration that refer to acts of varying degrees of severity. Descriptive statistics will describe what types of sexually violent acts occurred most frequently. Additionally, participants who indicated that they experienced or perpetrated an act of sexual harassment or sexual violence will be asked about the location of incident. Descriptive statistics will show where these acts have predominately occurred over the past year.

\section{Missing data and non-response}

Missing responses on the survey will be addressed through multiple imputation by chained equations (approximately 20 imputations based on best practice). ${ }^{23}$ Non-response bias will be tested through accessing basic sociodemographic data from the University of Oxford on the total population. A sensitivity analyses will be used to see if non-response is significantly associated with gender, year at university, and race, and to assess if responses need to be reweighted based given available information on the entire population demographic. Specifically, it may be the case that a representative subsample of responses will be selected for the analysis.

Analyses will be two-sided and use a 0.05 alpha to determine statistical significance. The Bonferroni correction will be applied to help counteract the issue of multiple 
testing. All statistical analyses will be conducted in $\mathrm{R}$ (V.3.5.1).

\section{DISCUSSION AND IMPLICATIONS}

Sexual violence in general, and in the HEI environment, has historically been unacknowledged or hidden. Victims under report sexual violence to official bodies and when they do, there is evidence that such instances have been insufficiently dealt with. ${ }^{24}$ Survey data play a crucial role in discerning a more accurate prevalence rate and allowing student voices to shape the communities in which they live and study and inform the prevention and response practices delivered by their institutions. ${ }^{25}$

Currently, there are limited data in the UK to inform UK HEIs on how to prevent and respond to sexual violence. This study has the potential to impact the way practitioners and researchers understand the problem of sexual violence at UK HEIs, inform the development of evidence-based programming to address the issue of sexual violence, and evaluate the effectiveness of preexisting supports on campus. This research project is a collaboration with the University of Oxford and implements a new methodology for tracking sexual violence in the UK context. The long-term goal of this survey is to form a template that can be used and adapted by other UK universities to evaluate sexual violence at their own institutions. These data will enable policy makers and Universities UK to make evidence-informed decisions about funding for sexual violence prevention and response, to assist officials in monitoring the issue, and to contribute to the investigation of cultures around systemic gender inequality within UK HEIs.

\section{LIMITATIONS}

A key limitation of this study is its use of a cross-sectional survey. As a consequence, it is not possible to establish causal links between risk and protective factor and the outcome of sexual violence or harassment. This survey, does however, have the potential to become a repeated cross-sectional survey given the transparent and reproducible research practices used and the existing partnerships and interest from university administration and student groups. Further, given that this study is the first of its kind in the UK context, prevalence estimates can play an important first step in establishing a larger research agenda.

Another key limitation of this study is the likely presence of response bias-the potential for certain groups of people to be more likely to respond to the survey compared with others. Although we assume in our power calculation that a random selection of the population will respond, this will likely not be the case. Participants may be more likely to respond if they have experienced sexual harassment or sexual violence. Alternatively, participants who have experienced sexual harassment or sexual violence may be deterred from responding to prevent retraumatisation. To help mitigate the issue of response bias we will weight certain survey variables based on known total population characteristics. ${ }^{26}$

A further potential limitation is that this study is being administered during the COVID-19 pandemic and therefore, the interpretation of prevalence rates must consider that the living arrangements and social activities of participants during the reporting period would have been impacted by government and university safety regulations. As a result, we have incorporated questions on online sexual harassment which helps to capture the potentially increasing relationship between social media and sexual harassment during the pandemic.

\section{ETHICS AND DISSEMINATION}

This research will take careful consideration of ethics and confidentiality as dictated by the University of Oxford and will adhere to high quality research practices. All aspects of this research have obtained approval from the University of Oxford (ref no.: R73805/RE001). Participants will be provided with a consent and information form when they receive the electronic survey. Students will be able to provide digital consent after reading this form by ticking a box and agreeing to proceed with the survey. The consent and information forms indicate that responses to the survey will be confidential. Participant responses will be deidentified and given a unique alphanumerical study identifier for analysis. The Qualtrics survey platform ensures that responses are encrypted and only one member of the research team will be able to see email addresses. The research team will have single sign-on/ password-protected Qualtrics accounts.

Students will be informed of the sensitive nature of survey questions in the consent and information form. Each page of the survey will provide the contact information for sexual violence response support services both internal to the University of Oxford and external to the university. For example, students will be given information on services offered by the city of Oxford and by the police. The survey is short in duration and utilises skip logic so students will only be asked questions relevant to them and their experiences. Students will be told that they are under no obligation to complete the survey and that they can skip any response at any point during the survey to help prevent retraumatising victims.

The findings of this research will be presented at conferences and disseminated through peer-reviewed publications as well as a report to the student body. A key part of the knowledge translation process will involve the stakeholder groups who will inform best ways to ensure students and university administrators receive the information in a clear and transparent way. Up-to-date information on the survey and its results will be housed on the survey webpage.

Twitter Michelle Degli Esposti @Michelle_Degli 
Acknowledgements Thank you to Dr. Jacquelyn Campbell and Dr. Bushra Sabri for providing a copy of the Johns Hopkins University Health and Safety Study.

Contributors BS, DKH, PM, GH and EN conceived of the study idea. BS wrote the protocol with input from all authors. All authors contributed to the development of the survey tool. MDE provided input into the methodology and analysis plan. All authors approved the final protocol manuscript. DKH is the supervisor of the study. Funding This work was supported by the University of Oxford's van Houten fund. Competing interests PM is the Service Lead for the Sexual Harassment and Violence Support Service at the University of Oxford.

Patient and public involvement Patients and/or the public were involved in the design, or conduct, or reporting, or dissemination plans of this research. Refer to the Methods section for further details.

Patient consent for publication Not applicable.

Ethics approval Ethical approval was obtained by the Social Sciences and Humanities Interdivisional Research Ethics Committee at the University of Oxford which is a subcommittee of the Central University Research Ethics Committee (Ref No: R73805/RE001).

Provenance and peer review Not commissioned; externally peer reviewed.

Open access This is an open access article distributed in accordance with the Creative Commons Attribution Non Commercial (CC BY-NC 4.0) license, which permits others to distribute, remix, adapt, build upon this work non-commercially, and license their derivative works on different terms, provided the original work is properly cited, appropriate credit is given, any changes made indicated, and the use is non-commercial. See: http://creativecommons.org/licenses/by-nc/4.0/.

ORCID iDs

Bridget Steele http://orcid.org/0000-0002-2028-558X

Michelle Degli Esposti http://orcid.org/0000-0002-0068-5754

\section{REFERENCES}

1 Krebs CP, Lindquist CH, Warner TD. The campus sexual assault (CsA) study. Washington, DC: National Institute of Justice, US Department of Justice, 2007.

2 DeGue S, Valle LA, Holt MK, et al. A systematic review of primary prevention strategies for sexual violence perpetration. Aggress Violent Behav 2014;19:346-62.

3 Krause KH, Woofter R, Haardörfer R, et al. Measuring campus sexual assault and culture: a systematic review of campus climate surveys. Psychol Violence 2019;9:611-22.

4 Sinozich S, Langton L. Rape and sexual assault among college-age females, 1995-2013. BJS Special Report 2014.

5 Fedina L, Holmes JL, Backes BL. Campus sexual assault: a systematic review of prevalence research from 2000 to 2015. Trauma Violence Abuse 2018;19:76-93.
6 Cantor D, Fisher B, Chibnall SH. Report on the AAU campus climate survey on sexual assault and sexual misconduct, 2015.

7 Harris AJ, Terry KJ, Ackerman AR. Campus sexual assault: forging an Action-Focused research agenda. Sex Abuse 2019;31:263-9.

8 Goldhill O, Bingham J. One in three UK female students sexually assaulted or abused on campus. The Telegraph, 2015.

9 Batty D, Weale S, Bannock C. Sexual harassment 'at epidemic levels' in UK universities. The Guardian 2017;5:2017.

10 Smith G. Hidden marks: A study of women students' experiences of harassment, stalking, violence and sexual assault, 2010.

11 UK U. Changing the culture: report of the universities UK Taskforce examining violence against women, harassment and hate crime affecting university students, 2016.

12 Sweeting H, Thomson H, Wells V. Evolution of 'whole institution' approaches to improving health in tertiary education settings: a critical scoping review. Res Pap Educ 2021;25:1-29.

13 Basile KC, Smith SG, Breiding M. Sexual violence surveillance: uniform definitions and recommended data elements. version 2.0, 2014.

14 About: University of Oxford, 2020. Available: https://www.ox.ac.uk/ about/organisation?wssl=1 [Accessed 07 Jul 2020].

15 Facts and figures: University of Oxford, 2020. Available: https://www. ox.ac.uk/about/facts-and-figures/full-version-facts-and-figures? wssl $=1$ [Accessed 07 Jul 2020].

16 Koss MP, Abbey A, Campbell R, et al. Revising the Ses: a collaborative process to improve assessment of sexual aggression and victimization. Psychol Women Q 2007;31:357-70.

17 Johnson SM, Murphy MJ, Gidycz CA. Reliability and validity of the sexual experiences Survey-Short forms victimization and Perpetration. Violence Vict 2017;32:78-92.

18 Campbell JC, Sabri B, Budhathoki C, et al. Unwanted sexual acts among university students: correlates of victimization and Perpetration. J Interpers Violence 2021;36:NP504-26.

19 Luetke M, Giroux S, Herbenick D, et al. High prevalence of sexual assault victimization experiences among university Fraternity men. $J$ Interpers Violence 2020:088626051990028.

20 Krebs C, Lindquist C, Berzofsky M. Campus climate survey validation study: final technical report. BJS, Office of Justice Programs, 2016.

21 Hajian-Tilaki K. Sample size estimation in diagnostic test studies of biomedical informatics. J Biomed Inform 2014;48:193-204.

22 Spronk I, Korevaar JC, Poos R, et al. Calculating incidence rates and prevalence proportions: not as simple as it seems. BMC Public Health 2019;19:1-9.

23 Little RJ, Rubin DB. Statistical analysis with missing data. John Wiley \& Sons, 2019.

24 Wooten SC. HETEROSEXIST discourses how feminist theory shaped campus sexual violence policy, 2016.

25 Godenzi A, Schwartz MD, Dekeseredy WS. Toward a gendered social bond/male peer support theory of university woman abuse. Crit Criminol 2001;10:1-16.

26 Buskirk TD, Kolenikov S. Finding respondents in the forest: a comparison of logistic regression and random forest models for response propensity weighting and stratification. survey methods: insights from the field, 2015: 1-17. 